Cholesteric and screw-like nematic phases in systems of helical particles

Giorgio Cinacchi, Alberta Ferrarini, Achille Giacometti, and Hima Bindu Kolli

Citation: The Journal of Chemical Physics 147, 224903 (2017);

View online: https://doi.org/10.1063/1.4996610

View Table of Contents: http://aip.scitation.org/toc/jcp/147/22

Published by the American Institute of Physics

AIP| The Jouminal Pal Phyics

PERSPECTIVES 


\title{
Cholesteric and screw-like nematic phases in systems of helical particles
}

\author{
Giorgio Cinacchi, ${ }^{1, a)}$ Alberta Ferrarini, ${ }^{2, b)}$ Achille Giacometti, ${ }^{3, c)}$ and Hima Bindu Kolli ${ }^{4}$ \\ ${ }^{1}$ Departamento de Física Teórica de la Materia Condensada, Instituto de Física de la Materia Condensada \\ (IFIMAC) and Instituto de Ciencias de Materiales "Nicolás Cabrera", Universidad Autónoma de Madrid, \\ Campus de Cantoblanco, 28049 Madrid, Spain \\ ${ }^{2}$ Dipartimento di Scienze Chimiche, Università di Padova, Via F. Marzolo 1, 35131 Padova, Italy \\ ${ }^{3}$ Dipartimento di Scienze Molecolari e Nanosistemi, Università Ca' Foscari di Venezia, Via Torino 155, \\ 30172 Venezia Mestre, Italy \\ ${ }^{4}$ Hylleraas Centre for Quantum Molecular Sciences, Department of Chemistry, University of Oslo, \\ Postboks 1033 Blindern, 0315 Oslo, Norway
}

(Received 18 July 2017; accepted 13 November 2017; published online 8 December 2017)

\begin{abstract}
Recent numerical simulations of hard helical particle systems unveiled the existence of a novel chiral nematic phase, termed screw-like, characterised by the helical organization of the particle $\mathrm{C}_{2}$ symmetry axes round the nematic director with periodicity equal to the particle pitch. This phase forms at high density and can follow a less dense uniform nematic phase, with relative occurrence of the two phases depending on the helix morphology. Since these numerical simulations were conducted under threedimensional periodic boundary conditions, two questions could remain open. First, the real nature of the lower density nematic phase, expected to be cholesteric. Second, the influence that the latter, once allowed to form, may have on the existence and stability of the screw-like nematic phase. To address these questions, we have performed Monte Carlo and molecular dynamics numerical simulations of helical particle systems confined between two parallel repulsive walls. We have found that the removal of the periodicity constraint along one direction allows a relatively-long-pitch cholesteric phase to form, in lieu of the uniform nematic phase, with helical axis perpendicular to the walls while the existence and stability of the screw-like nematic phase are not appreciably affected by this change of boundary conditions. Published by AIP Publishing. https://doi.org/10.1063/1.4996610
\end{abstract}

\section{INTRODUCTION}

The propagation of chirality from the microscopic to the macroscopic scale is an issue of importance, both for fundamental science and potential applications. One very interesting case is represented by chiral particles experiencing only steric interactions. Within this class, one of the most natural and simplest models is the hard helix. More than 40 years ago, ${ }^{1}$ hard helices of sufficiently high aspect ratio were predicted to form a cholesteric $\left(\mathrm{N}_{c}^{*}\right)$ phase, a nematic liquid-crystal ${ }^{2}$ phase in which the main (usually long) particle axes are locally preferentially aligned parallel to one another and the average alignment axis, the nematic director $(\widehat{n})$, revolves in a helical fashion round a perpendicular axis $(\widehat{\mathbf{h}})$ with a halfpitch $(\mathcal{P} / 2)$ a few orders of magnitude larger than any particle dimension. Within the same framework, ${ }^{1}$ a relationship was proposed between the handedness of the $\mathrm{N}_{c}^{*}$ phase and the morphology (not simply the handedness) of the constituent helices. More recently, we addressed the phase behaviour of hard helical particles by Onsager-like (density functional) theory and Monte Carlo numerical simulation. ${ }^{3-7}$ We found a rich liquid-crystal polymorphism in terms of the helix morphology. Particularly noteworthy was the observation of a novel chiral nematic phase, termed screw-like $\left(\mathrm{N}_{s}^{*}\right)$, distinct from the $\mathrm{N}_{c}^{*}$

\footnotetext{
a)Electronic mail: giorgio.cinacchi@uam.es

b)Electronic mail: alberta.ferrarini@unipd.it

c)Electronic mail: achille.giacometti@unive.it
}

phase. In the $\mathrm{N}_{s}^{*}$ phase, $\widehat{n} \| \widehat{\mathbf{h}}$, and it is a transverse director $(\widehat{\mathbf{m}})$ that revolves in a helical fashion round $\widehat{\mathbf{h}}$ with a pitch equal to that of the particle. While the $\mathrm{N}_{c}^{*}$ phase can be exhibited by any non-racemic system of chiral nematogenic particles, the $\mathrm{N}_{s}^{*}$ phase is special to helical particles, and its formation sensitively depends on the helix morphology. It is this $\mathrm{N}_{s}^{*}$ phase that was observed in experiments on colloidal suspensions of helical flagella, ${ }^{8}$ and its possible existence in dense DNA solutions was also interestingly hypothesized. ${ }^{9}$

In our previous numerical simulations, ${ }^{3-7}$ threedimensional periodic boundary conditions (3D-PBC) were used. While entirely compatible with the $\mathrm{N}_{s}^{*}$ phase, 3D-PBC can clearly be inadequate in the case of a $\mathrm{N}_{c}^{*}$ phase with a value of $\mathcal{P} / 2$ orders of magnitude larger than computational box dimensions. ${ }^{10}$ In our numerical simulations, a uniform nematic $(\mathrm{N})$ phase was thus observed, which could precede the $\mathrm{N}_{s}^{*}$ phase on increasing density from the isotropic phase. Theoretical calculations made by us, $, 7,7$ reproduced by others, ${ }^{11-13}$ confirm the expectation ${ }^{1,3,4,6}$ that this $\mathrm{N}$ phase is actually cholesteric while providing a prediction for the sign and the value of the corresponding $\mathcal{P} .{ }^{14-17}$ This could raise the question of the actual observability of a $\mathrm{N}_{c}^{*}$ phase for helical particles together with that of the actual existence and stability of the $\mathrm{N}_{s}^{*}$ phase with respect to a change in the boundary conditions that would allow a proper $\mathrm{N}_{c}^{*}$ phase to form. ${ }^{18}$ The aim of the present study is to address both these points.

The numerical simulation of cholesteric liquid crystals poses specific problems. ${ }^{19}$ In the last few years, this 
challenging objective has been particularly pursued. ${ }^{20-22}$ Hard helical particles were marginally addressed in Ref. 21 where difficulties arising in this case, related to the narrow stability range of the $\mathrm{N}_{c}^{*}$ phase and the length of $\mathcal{P}$, were remarked.

Here, we further clarify the nature of the nematic phases formed by helical particles, removing any doubt about possible artefacts deriving by the use of 3D-PBC. By using two different independent numerical simulation methods, Monte Carlo and molecular dynamics, we will explicitly show the existence of a $\mathrm{N}_{c}^{*}$ phase for not-too-curly helical particles, thus supporting the suggestion ${ }^{3-7}$ that the previously observed $\mathrm{N}$ phase will actually turn cholesteric once 3D-PBC will have been suitably removed, as well as confirm the existence and stability of the $\mathrm{N}_{s}^{*}$ phase for sufficiently curly helical particles also under these other boundary conditions. ${ }^{23}$

In Sec. II, we will recall the model and describe the numerical simulation protocols. Section III presents the results of this study preceded by a summary of past results to cast it in the appropriate perspective. Section IV summarises the main findings.

\section{MODEL AND COMPUTATIONAL DETAILS}

In keeping with previous studies, ${ }^{3-7,24}$ the model particles considered are made of 15 partially overlapping hardor soft-repulsive spheres of diameter $D$, the unit of length, equidistantly and rigidly arranged along a right-handed helix of fixed contour length $L=10 D$, varying radius $r$ and pitch $p$ and whose long and short axes are, respectively, denoted as $\widehat{\mathbf{u}}$ and $\widehat{\mathbf{w}}$ (Fig. 1).

In case the spherical beads are soft-repulsive, two of them belonging to different particles interact via the repulsive part $V\left(r_{k l}\right)$ of the common separation of the Lennard-Jones potential energy function ${ }^{25}$

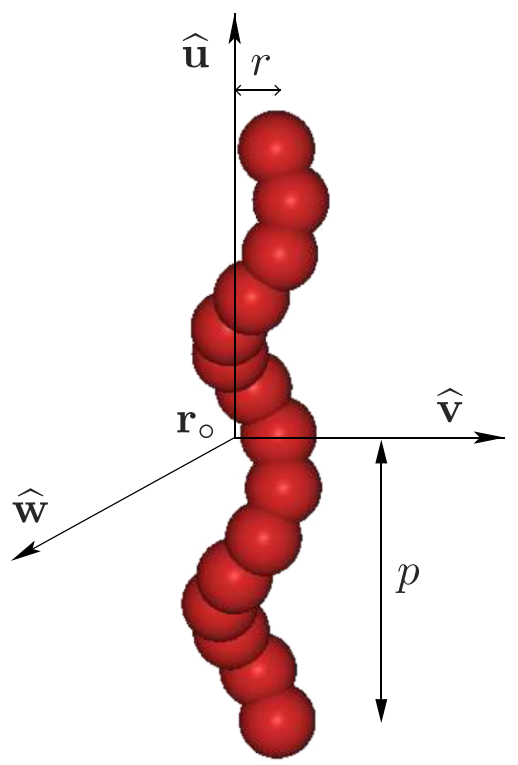

FIG. 1. Illustration of a particle composed of 15 partially overlapping spherical beads of diameter $D$ whose centres are equidistantly and rigidly arranged along a right-handed helical cord of length $L$, radius $r$, and pitch $p$. The mechanical state of any such helical particle can be defined by specifying its reference frame with respect to the laboratory reference frame: the position of the origin $\mathbf{r}_{\circ}$ and the orientation of the three mutually perpendicular unit vectors: $\widehat{\mathbf{u}}, \widehat{\mathbf{v}}$, and $\widehat{\mathbf{w}}$.

$$
V\left(r_{k l}\right)=\left\{\begin{array}{rl}
4 \epsilon\left[\left(\frac{D}{r_{k l}}\right)^{12}-\left(\frac{D}{r_{k l}}\right)^{6}+\frac{1}{4}\right] & : r_{k l} \leq \sqrt[6]{2} D \\
0 \quad: r_{k l} & >\sqrt[6]{2} D
\end{array},\right.
$$

with $r_{k l}$ being the distance between the centres of beads $k$ and $l$ and $\epsilon$ being the unit of energy.

For an on-lattice model, self-determined spiralling boundary conditions were devised as the solution to the problem of dealing with phases whose structure is characterised by a length scale that may be incommensurate to the dimensions of the lattice. ${ }^{26,27}$ In the same remarkable work, ${ }^{26}$ it was stated that the next best solution to this problem would be to employ free boundary conditions. For an off-lattice model, self-determined spiralling boundary conditions cannot be properly employed. Then, we have followed the suggestion $^{20}$ that a $\mathrm{N}_{c}^{*}$ phase can be investigated in a numerical simulation by confining the system between two suitable $\widehat{\mathbf{h}}$-perpendicular walls. The price to pay is to deal with a confined rather than bulk system. However, if the slab is sufficiently thick, the properties of the confined system sufficiently far away from the walls are equivalent to the corresponding properties of the bulk system.

Thus, the adopted computational protocol consists of two parts. First, it is observed that confining between two parallel hard- or soft-repulsive walls a sufficiently large and potentially cholesterogenic system, previously studied under 3D-PBC and seen to be nematogenic, does not alter its properties away from the walls; it only makes $\widehat{n}$ twist and a $\mathrm{N}_{c}^{*}$ phase result. Second, it is observed that the $\mathrm{N}_{s}^{*}$ phase either remains stable even under confinement, when a $\mathrm{N}_{c}^{*}$ phase could form, or spontaneously develops when starting from a cholesteric configuration.

(Enantiomerically) pure systems of $N \in[1500,5000]$ (freely translating and rotating) hard- or soft-repulsive helical particles, identified by the pair $(r, p)$, were placed between two fixed parallel, respectively, hard- or soft-repulsive, flat walls perpendicular to the $x$ axis of the laboratory reference frame. In case of soft-repulsive walls, their interactions with a spherical bead $k$ were described via the potential energy function $W_{9 / 3}\left(r_{k}\right)$

$$
W_{9 / 3}\left(r_{k}\right)= \begin{cases}\epsilon\left[\frac{2}{15}\left(\frac{D}{r_{k}}\right)^{9}-\left(\frac{D}{r_{k}}\right)^{3}+1\right] & : r_{k} \leq \sqrt[6]{0.4} D \\ 0 & : r_{k}>\sqrt[6]{0.4} D\end{cases}
$$

with $r_{k}$ being the distance of the centre of the spherical bead $k$ from a wall. Such helical-particle-wall interactions promote planar alignment of the helical particles close to the walls so that $\widehat{\mathbf{h}} \| x$.

The systems were investigated via, respectively, the Monte Carlo (MC) method ${ }^{28}$ or the molecular dynamics (MD) method. ${ }^{29}$ Both were run in the isobaric-isothermal (NPT) ensemble $\mathrm{e}^{30-32}$ for several values of pressure $\mathrm{P}$ measured in units $k_{B} \mathrm{~T} / D^{3}$, with $k_{B}$ being the Boltzmann constant and $\mathrm{T}$ being the absolute temperature, in the MC case and in units $\epsilon / D^{3}$, with $k_{B} \mathrm{~T} / \epsilon=1$, in the MD case. Both the MC-NPT and MD-NPT calculations were carried out under rectangular periodic boundary conditions along the $y$ and $z$ axes of the laboratory reference frame. ${ }^{20}$ The MC-NPT calculations were organised in cycles, each consisting of $2 \mathrm{~N}$ attempts to translate or rotate a randomly selected helical particle plus an attempt to change the cuboidal computational box shape and volume 

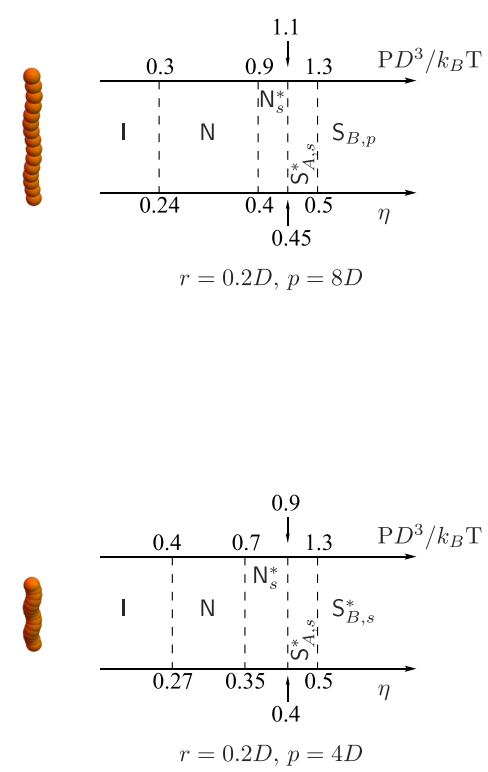
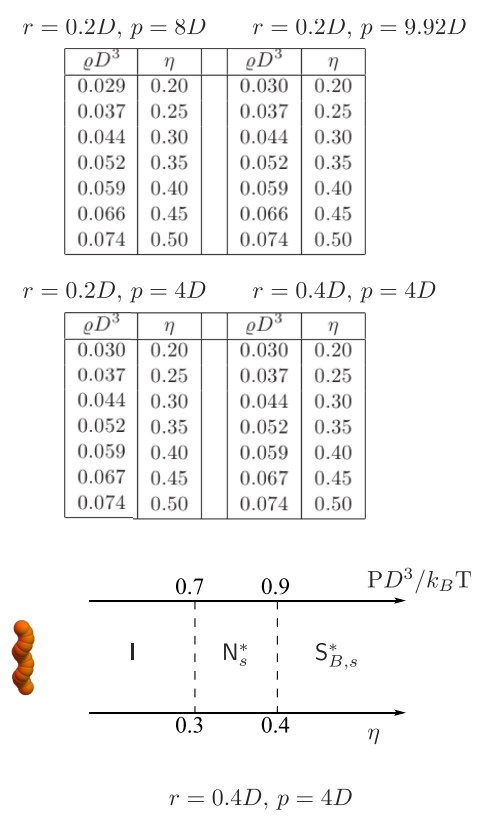

FIG. 2. Summary of the phase diagrams, obtained from numerical simulations with 3D-PBC, for three different helix morphologies, as a function of the volume fraction $\eta=\varrho v$, with $v$ being the volume of a helical particle whose image is included: (top left) $r=0.2 D$ and $p=8 D$; (bottom left) $r=0.2 D$ and $p=4 D$; (bottom right) $r=0.4 D$ and $p=4 D$. Here, $I=$ isotropic phase, $\mathrm{N}=$ uniform nematic, $\mathrm{N}_{s}^{*}=$ screw-like nematic, $\mathrm{S}_{A, s}^{*}$ $=$ screw-like smectic $\mathrm{A}$, and $\mathrm{S}_{B, s}^{*}=$ screw-like smectic B. $\mathrm{S}_{B, p}=$ polar smectic B. The relations between $\eta$ and $\varrho D^{3}$ for these three cases as well as the case with $r=0.2 D$ and $p=9.92 D$ are explicitly given (top right). by independently changing the length of its $L_{y}$ or $L_{z}$ edge. ${ }^{20}$ The MD-NPT calculations were carried out with the program LAMMPS, ${ }^{33}$ integrating the equations of motion via a rigidparticle algorithm ${ }^{34}$ with a time step $\tau=0.01 \sqrt{m D^{2} / \epsilon}$, with $m$ being the mass of a spherical bead, and using a semi-isotropic barostat, allowing the cuboidal computational box to fluctuate in the $y z$ plane, and a thermostat whose damping parameters to control pressure and temperature, $\tau_{\mathrm{P}}$ and $\tau_{\mathrm{T}}$, were both equal to $\sqrt{m D^{2} / \epsilon}$. Both MC-NPT and MD-NPT calculations were started: (i) either from a configuration equilibrated during previous numerical simulations and inserted between the two parallel hard- or soft-repulsive walls taking care to remove those particles that happened to overlap with them; (ii) or a moderately dense orthorhombic lattice configuration with all helical particle $\widehat{\mathbf{u}}$ axes perfectly aligned along the $z$ axis; (iii) or a configuration obtained as an output of a run at a nearby value of pressure P. Rather lengthy simulations had to be employed. In the MC case, most runs were of 15 or more million MC cycles. In the MD case, the trajectories were up to $320 \times 10^{6}$ time step long.

In the (production) runs, averages of several quantities were accumulated and configurations were stored for the subsequent analysis. The calculated quantities include the number density, $\varrho$, the nematic order parameter, ${ }^{35} S_{2}$, and the angle, $\theta$, that the local nematic director, $\widehat{n}(x)$, forms with the $y$ axis, as a function of $x$, along with a few suitable pair correlation functions to further ascertain the modulated-nematic nature of a phase. From a linear fit of $\theta(x)$, whose slope coincides with $q=2 \pi / \mathcal{P}$, an estimate of $\mathcal{P}$ was obtained. One pair correlation function is

$$
g_{2}^{\widehat{\mathbf{u}}}(x)=\left\langle\frac{\sum_{i=1}^{\mathrm{N}} \sum_{j>i}^{\mathrm{N}} P_{2}\left(\widehat{\mathbf{u}}_{i} \cdot \widehat{\mathbf{u}}_{j}\right) \delta\left(x-x_{i j}\right)}{\sum_{i=1}^{\mathrm{N}} \sum_{j>i}^{\mathrm{N}} \delta\left(x-x_{i j}\right)}\right\rangle,
$$

with $P_{n}()$ being the $n$ th-order Legendre polynomial, $\delta()$ being the standard $\delta$-function, $x_{i j}$ being the distance between particle $i$ and $j, \mathbf{r}_{i j}$, resolved along the $x$ axis, and \langle\rangle representing an average over configurations. This function is expected to behave as $S_{2}^{2} P_{2}(\cos (q x))$ if a cholesteric ordering is present and to be essentially flat otherwise. One more correlation function is $g_{1}^{\widehat{\mathbf{w}}}\left(r_{\|}, x\right)$, the suitable generalisation of the pair correlation function $g_{1}^{\widehat{\mathbf{w}}}\left(r_{\|}\right)$previously defined. ${ }^{4,6}$ Rather than explicitly considering this two-variable function, it proves convenient to consider either the function $G_{1}^{\widehat{\mathbf{w}}}(x)=g_{1}^{\widehat{\mathbf{w}}}(0, x) \equiv \max _{r_{\|}} g_{1}^{\widehat{\mathbf{w}}}\left(r_{\|}, x\right)$ or the function $g_{1, \Delta}^{\widehat{\mathbf{w}}, \widehat{\mathbf{u}}}\left(r_{/ /}\right)$ defined as

$$
g_{1, \Delta}^{\widehat{\mathbf{w}}, \widehat{\mathbf{u}}}\left(r_{/ /}\right)=\left\langle\frac{\sum_{i=1}^{\mathrm{N}} \sum_{j>i}^{\mathrm{N}} P_{1}\left(\widehat{\mathbf{w}}_{i} \cdot \widehat{\mathbf{w}}_{j}\right)\left[\left(x_{i j}-\Delta\right)<0\right] \delta\left(r_{/ /}-\mathbf{r}_{i j} \cdot \widehat{\mathbf{u}}_{i}\right)}{\sum_{i=1}^{\mathrm{N}} \sum_{j>i}^{\mathrm{N}}\left[\left(x_{i j}-\Delta\right)<0\right] \delta\left(r_{/ /}-\mathbf{r}_{i j} \cdot \widehat{\mathbf{u}}_{i}\right)}\right\rangle,
$$

with $\left[\left(x_{i j}-\Delta\right)<0\right]$ being an Iverson bracket. This variant of $g_{1}^{\widehat{\mathbf{w}}}\left(r_{\|}\right)$is expected to develop a well-defined $p$-periodic cosinusoidal form if a screw-like ordering is overall present and to be essentially flat otherwise. In case $g_{1, \Delta}^{\widehat{\mathbf{w}}, \widehat{\mathbf{u}}}\left(r_{/ /}\right)$reveals a presence of screw-like ordering, the function $G_{1}^{\widehat{\mathrm{w}}}(x)$ can further specify its dependence on $x$. Together, the pair correlation function $g_{2}^{\widehat{\mathbf{u}}}(x)$ and the duet $G_{1}^{\widehat{\mathbf{w}}}(x)-g_{1, \Delta}^{\widehat{\mathbf{w}}, \widehat{\mathbf{u}}}\left(r_{/ /}\right)$reveal whether the two types of modulated-nematic ordering are present, either jointly or separately. 


\section{RESULTS}

\section{A. Systems under investigation}

The present work builds upon past extensive numerical simulations, employing 3D-PBC, of systems of hard helical particles with various parameters $(r, p){ }^{3,4,6,7}$ Here, we have focused on three representative cases which were found to exhibit different propensity to form $\mathrm{N}$ and $\mathrm{N}_{s}^{*}$ phases, as schematically summarised in Fig. 2.

For helical particles with small $r$ and relatively long $p$ $(r=0.2 D$ and $p=8 D$ ), a broad-range $\mathrm{N}$ phase was found followed, at a progressively higher density, by a $\mathrm{N}_{s}^{*}$ phase, on a very narrow region, and screw-like smectic $\mathrm{A}$ and $\mathrm{B}$ phases. For helical particles with the same $r$ and a smaller $p(r=0.2 D$ and $p=4 D$ ), we found both $\mathrm{N}$ and $\mathrm{N}_{s}^{*}$ phases to be present in respective regions of comparable width. For helical particles
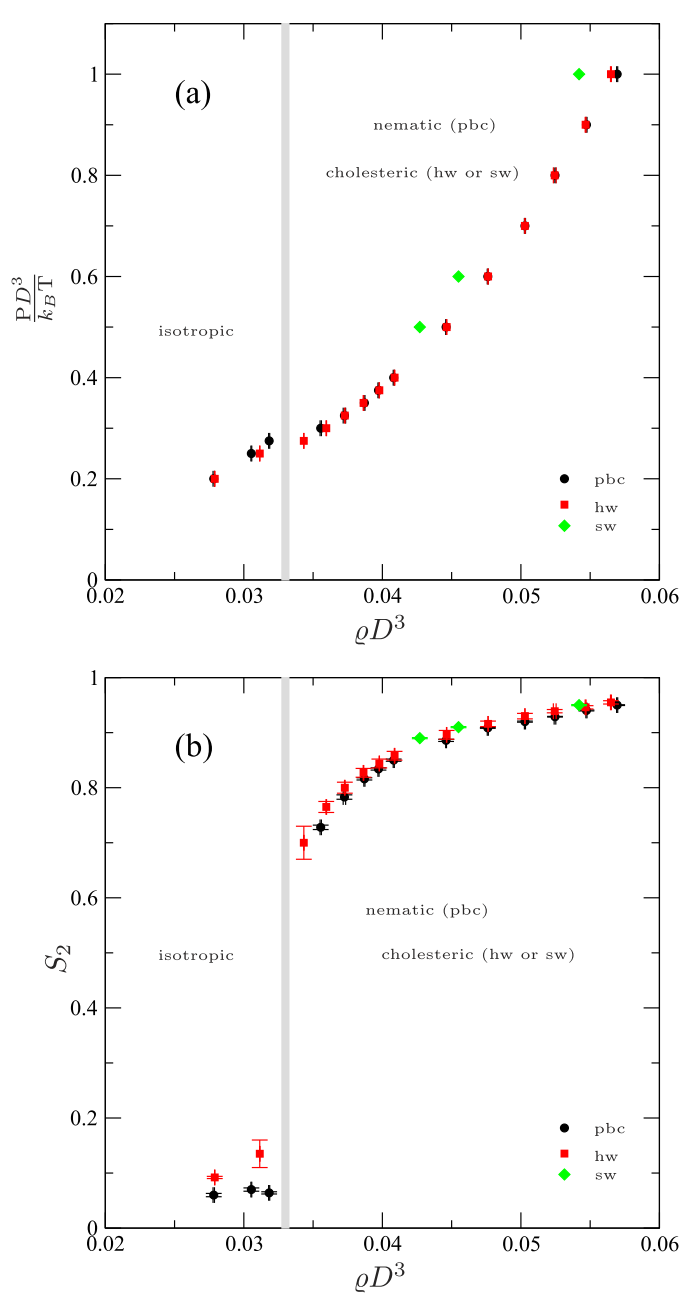

FIG. 3. Behaviour of dimensionless pressure $\frac{\mathrm{P} D^{3}}{k_{B} \mathrm{~T}}$ (a) and nematic order parameter $S_{2}$ (b) as a function of dimensionless number density $\varrho D^{3}$ for a system of hard helical particles with $r=0.2 D$ and $p=9.92 D$ as obtained in MC numerical simulations employing 3D-PBC (pbc, black circles) or confining the system between two parallel hard walls (hw, red squares). While in the former case, a uniform nematic phase is formed; in the latter, a cholesteric phase is observed. For comparison, a few data obtained for a system of softrepulsive helical particles confined between two parallel soft-repulsive walls (sw, green diamonds) which forms a cholesteric phase have been also included. In both panels, the gray slender box delimits either of these nematic phases and the coexistent isotropic phase. with a larger $r$ and the same $p(r=0.4 D$ and $p=4 D)$, the $\mathrm{N}$ phase was absent and a region of $\mathrm{N}_{s}^{*}$ phase was found.

These findings were rationalized ${ }^{4,6}$ in terms of an entropy gain triggered by the coupling between the translation of the particles along $\widehat{n}$ and rotation round their own $\widehat{\mathbf{u}}$. On increasing their curliness, neighbouring parallel helical particles tend to mutually interlock their grooves, thus restraining rotation round their $\widehat{\mathbf{u}}$. This rotational entropy loss can be compensated by a translational motion along $\widehat{n}$ in a way similar to a screw, hence the name given to the new chiral nematic phase.

\section{B. $r=0.2 D$ and $p=9.92 D$}

The examination of the obtained results starts with those for systems of helical particles having $r=0.2 D$ and $p=9.92 D$. This value of $p$ is such that for a contour length $L$, it corresponds to a single helical turn. These helical particles, nearly straight rods, have a wider $\mathrm{N}$ phase as compared to the $p=8 D$ counterpart (Fig. 2) and are expected to form the $\mathrm{N}_{c}^{*}$ phase only. ${ }^{3,4,6,7,36}$ In order to confirm this, Fig. 3 reports the equation of state (EoS) and the density dependence of $S_{2}$ as obtained for a bulk system of hard helical particles
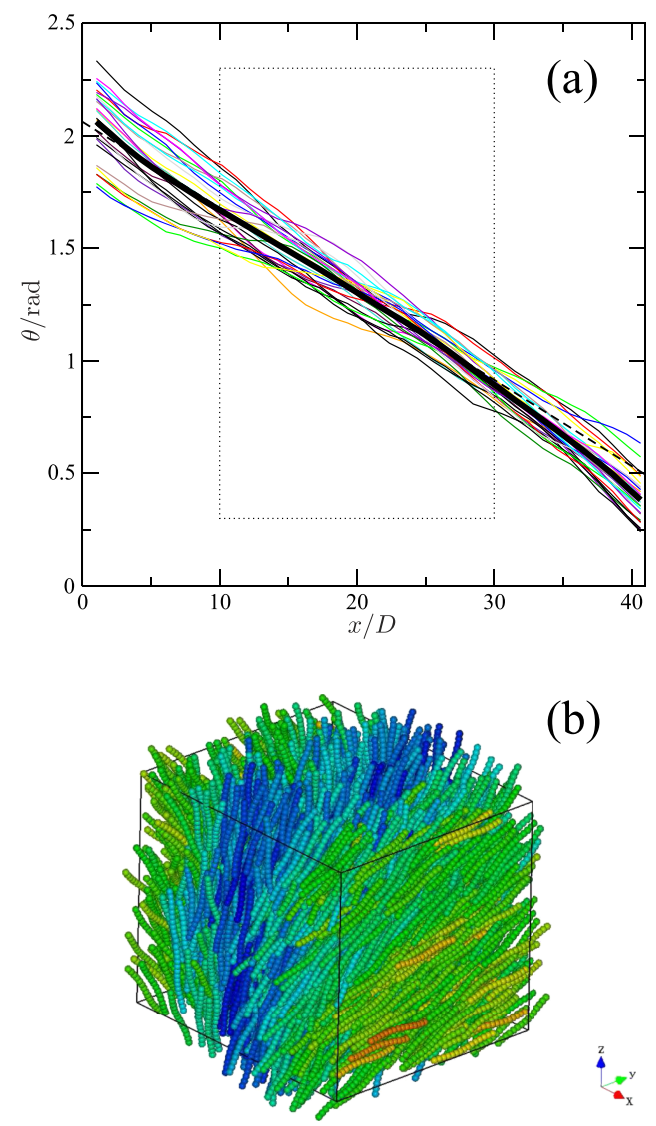

FIG. 4. (a) Behaviour of the angle $\theta$ that the local nematic director forms with the $y$ axis as a function of $x$ for a system of hard helical particles with $r=0.2 D$ and $p=9.92 D$ at $\mathrm{P}^{3} / k_{B} \mathrm{~T}=0.5$. The various coloured thin full curves shown are each an average over 200, one-thousand-MC-cycle separated, configurations while the black thick full curve is their global average and the black thick dashed line is the linear fit to the latter taking into account only the region enclosed by the dotted rectangle. (b) Image ${ }^{37}$ of the cholesteric phase for a system of hard helical particles with $r=0.2 D$ and $p=9.92 D$ at $\mathrm{PD}^{3} / k_{B} \mathrm{~T}$ $=0.5$. The hard helical particles are coloured according to the angle that their $\widehat{\mathbf{u}}$ axes forms with the eigenvector corresponding to the largest eigenvalue of the global nematic order matrix. ${ }^{35}$ 

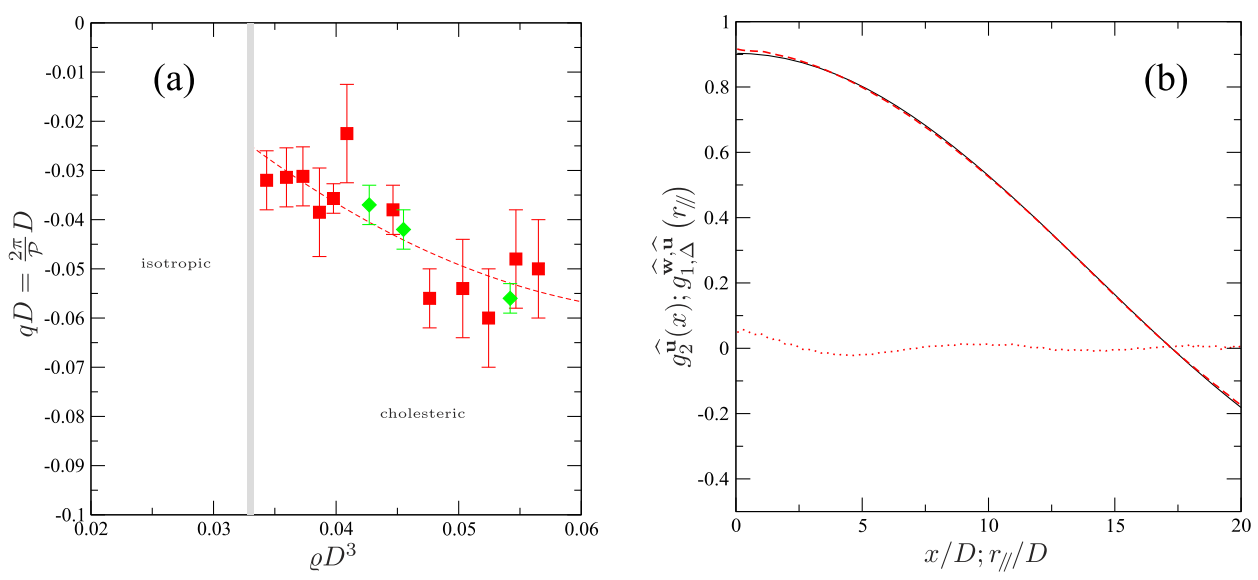

FIG. 5. (a) Behaviour of dimensionless wavevector $q D=\frac{2 \pi}{\mathcal{P}} D$ as a function of dimensionless number density $\varrho D^{3}$ (red squares) for a system of hard helical particles with $r=0.2 D$ and $p=9.92 D$. The red dashed line is a quadratic fit to the data, meant as a mere guide to the eye. Included also are a few data obtained for a system of soft-repulsive helical particles confined between two parallel soft-repulsive walls (green diamonds). The gray slender box delimits the cholesteric phase and the coexistent isotropic phase. (b) pair correlation functions $g_{2}^{\widehat{\mathbf{u}}}(x)$ (red dashed line) and $g_{1, \Delta}^{\widehat{\mathbf{w}}, \widehat{\mathbf{u}}}\left(r_{/ /}\right)$(red dotted line) for a system of hard helical particles with $r=0.2 D$ and $p=9.92 D$ at $\mathrm{P} D^{3} / k_{B} \mathrm{~T}=1$. The black thinner full line is a fit of $g_{2}^{\widehat{\mathbf{u}}}(x)$ with the function $S_{2}^{2} P_{2}(\cos (q x))$ with $S_{2}=0.950$ and $|q| D=0.0554$ as fitting parameters, consistent with, respectively, the value of $S_{2}$ in Fig. 3(b) and the absolute value of $q D$ in (a).

employing 3D-PBC and for a system of the same particles confined between two parallel hard walls. Included are also a few data obtained for a system of soft-repulsive helical particles confined between two parallel soft-repulsive walls. In all cases, the average values of $S_{2}$ correspond to the average values of this quantity calculated by discarding the contribution of particles closer than $10 D$ to either wall. The choice of this distance had been made by plotting the number density $\varrho(x)$ and nematic order parameter $S_{2}(x)$ profiles with respect to $x$, and by observing that, farther than that distance, confinement effects had reasonably faded away. From Fig. 3, it can be seen that: (i) the isotropicnematic (cholesteric) phase transition is slightly shifted to a lower value of density as compared to the case $r=0.2 D$ and $p=8 D$, as expected in view of the increased aspect ratio of the helical particles and (ii) both the EoS and $S_{2}$ versus $\varrho$ graphs for the confined system match the corresponding graphs for the bulk system. However, the latter is in the $\mathrm{N}$ phase whereas the former relatively promptly forms a $\mathrm{N}_{c}^{*}$ phase. Figure 4 shows the behaviour of $\theta(x)$, calculated at $\mathrm{P} D^{3} / k_{B} \mathrm{~T}=0.5$, along with an image of a configuration in the $\mathrm{N}_{c}^{*}$ phase at the same value of dimensionless pressure. Discarding the contribution of the slices closer than $10 D$ to either wall, linear fits to the various calculated $\theta(x)$ 's were performed. The values of $q$ thus estimated are given in Fig. 5(a) as a function of $\varrho$. The negative sign of $q$ indicates a left-handed $\mathrm{N}_{c}^{*}$ phase, as predicted by Straley ${ }^{1}$ and Onsager-like theory calculations $s^{5,7,11-13}$ for right-handed hard helical particles with geometric parameters analogous to those of the system under investigation. The large error bars reflect wide fluctuations of the local director which
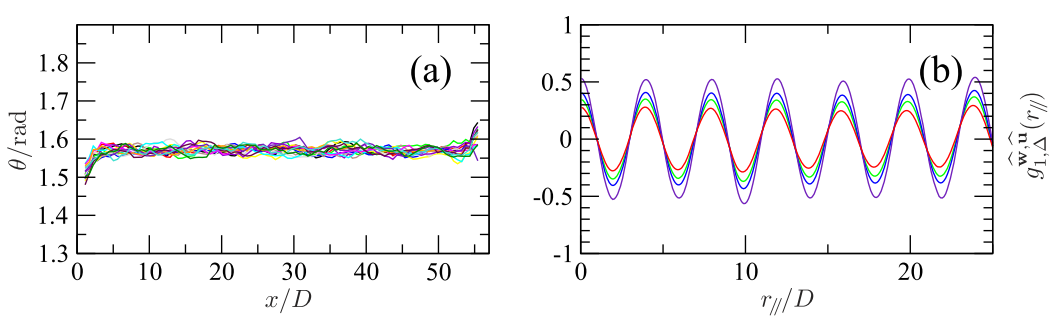

(c)
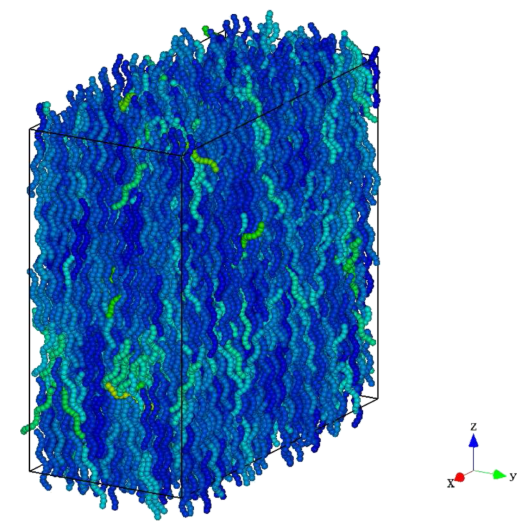

FIG. 6. For a system of hard helical particles with $r=0.4 D$ and $p=4 D$ : (a) the angle $\theta$ that the local nematic director forms with the $y$ axis as a function of $x$ at $\mathrm{P} D^{3} / k_{B} \mathrm{~T}=0.75$, the various coloured thin full curves shown being each an average over 1000, one-thousandMC-cycle separated, configurations; (b) the pair correlation function $g_{1, \Delta}^{\widehat{\mathbf{w}}, \widehat{\mathbf{u}}}\left(r_{/ /}\right)$at $\mathrm{P} D^{3} / k_{B} \mathrm{~T}=0.7,0.75,0.8,0.9$; (c) image $\mathrm{e}^{37}$ of the screw-like nematic phase at $\mathrm{P} D^{3} / k_{B} \mathrm{~T}$ $=0.75$. The hard helical particles are coloured according to the angle that their $\widehat{\mathbf{u}}$ axes forms with the eigenvector corresponding to the largest eigenvalue of the global nematic order matrix. ${ }^{35}$ 
do not allow a precise determination of $\mathcal{P} .{ }^{38}$ Nonetheless, the magnitude of $q$, corresponding to $\mathcal{P}$ in the range of $100-200 D$, which decreases with increasing density, compares well to the theoretical calculations for hard helical particles with $r=0.2 D$ and $p=8 D$ in Ref. 7. The functions $g_{2}^{\widehat{\mathbf{u}}}(x)$ and $g_{1, \Delta}^{\widehat{\mathbf{w}}, \widehat{u}}\left(r_{/ /}\right)$at $\mathrm{P} D^{3} / k_{B} \mathrm{~T}=1$ are shown in Fig. 5(b). Their behaviour confirms the cholesteric character of the nematic phase and the expected absence of screw-like ordering [yet, an extremely tenuous short-range undulation is already visible in the function $\left.g_{1, \Delta}^{\widehat{\mathbf{w}}, \widehat{u}}\left(r_{/ /}\right)\right]$; these helical particles are not sufficiently curly to promote, at these values of $\varrho$, the setting in of the latter type of ordering.

\section{C. $r=0.4 D$ and $p=4 D$}

The case of curly helical particles with $r=0.4 D$ and $p=4 D$ stands at the other extreme. Their nematic phase is screw-like, existing in the interval $\mathrm{P} D^{3} / k_{B} \mathrm{~T} \in(0.7,0.9)$, bounded by a less dense isotropic phase and a denser screwlike smectic B phase. ${ }^{4,6,7}$ This phase sequence, previously obtained by employing 3D-PBC for systems of hard- ${ }^{4,6,7}$ and soft-repulsive ${ }^{24}$ helical particles with these values of $r$ and $p$, is confirmed by the present MC-NPT calculations carried out under confinement of two parallel hard walls. Starting from a previously obtained configuration carefully inserted in between the two parallel hard walls, the $\mathrm{N}_{s}^{*}$ phase remains

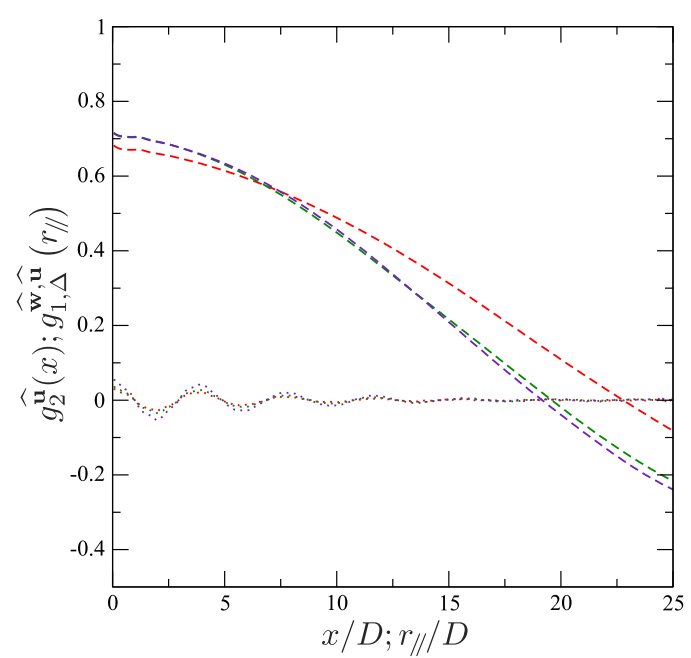

FIG. 7. Pair correlation functions $g_{2}^{\widehat{\mathbf{u}}}(x)$ (dashed line) and $g_{1, \Delta}^{\widehat{\mathbf{w}}, \widehat{\mathbf{u}}}\left(r_{/ /}\right)$(dotted line) for a system of hard helical particles with $r=0.2 D$ and $p=4 D$ at $\mathrm{P} D^{3} / k_{B} \mathrm{~T}=0.55$ from an initial nematic configuration (red); $\mathrm{P} D^{3} / k_{B} \mathrm{~T}=0.6$ from an initial nematic configuration (green); $\mathrm{P} D^{3} / k_{B} \mathrm{~T}=0.6$ from an initial configuration taken from the run at $\mathrm{P} D^{3} / k_{B} \mathrm{~T}=0.9$, in its turn started from an orthorhombic lattice configuration (indigo).

stable at $\mathrm{P} D^{3} / k_{B} \mathrm{~T}=0.7,0.8,0.9$ in the course of runs up to 7 million-MC cycle long (Fig. 6), while at $\mathrm{P} D^{3} / k_{B} \mathrm{~T}=0.6$, it transits directly to the isotropic phase.
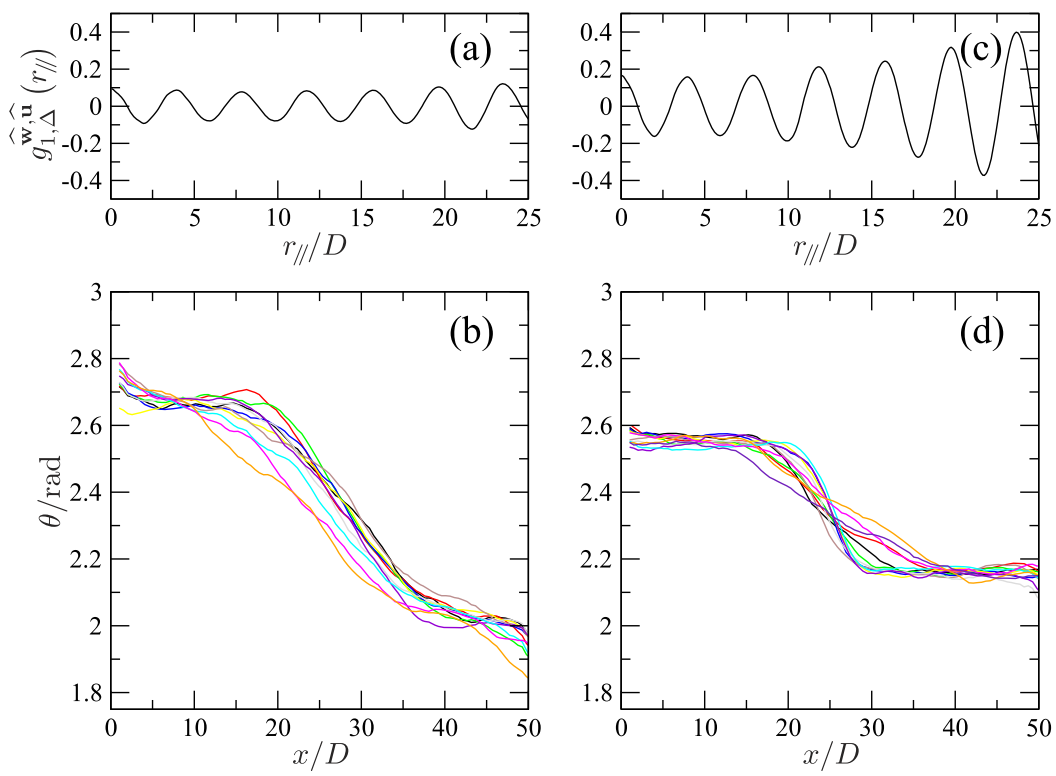

FIG. 8. Pair correlation functions $g_{1, \Delta}^{\widehat{\mathbf{w}}, \widehat{\mathbf{u}}}\left(r_{/ /}\right)$and the angle $\theta$ that the local nematic director form with the $y$ axis as a function of $x$ for a system of hard helical particles with $r$ $=0.2 D$ and $p=4 D$ as obtained in MC numerical simulations starting from a nematic configuration inserted between two parallel hard walls at $\mathrm{P} D^{3} / k_{B} \mathrm{~T}=0.7$ [(a) and (b)] and 0.8 [(c) and (d)]. The various coloured thin full curves shown in (b) and (d) are each an average over 1000 , one-thousand-MC-cycle separated, configurations. In (e), the function $G_{1}^{\widehat{\mathbf{w}}}(x)$ is also shown at $\mathrm{P}^{3} / k_{B} \mathrm{~T}=0.7$ (red) and $\mathrm{P} D^{3} / k_{B} \mathrm{~T}=0.8$ (green).

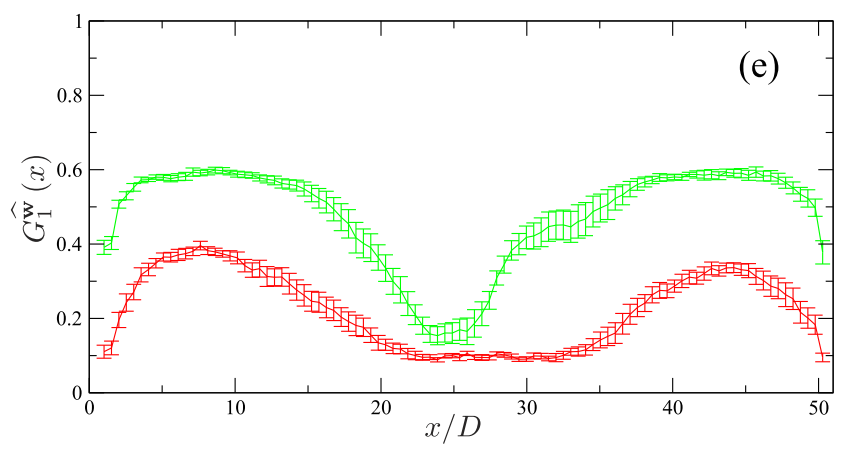




\section{D. $r=0.2 D$ and $p=4 D$}

The last representative case is given by helical particles with $r=0.2 D$ and $p=4 D$, where both cholesteric and screw-like orderings are present (Fig. 2). For these hard helical particle systems, a number of MC-NPT calculations were carried out at the following values of $\mathrm{P} D^{3} / k_{B} \mathrm{~T}$ : $0.55 ; 0.6 ; 0.7$; 0.8 ; and 0.9. In previous numerical simulations employing 3D$\mathrm{PBC}$, the state points at $\mathrm{P} D^{3} / k_{B} \mathrm{~T}=0.55$ and 0.6 were assigned to a $\mathrm{N}$ phase. Starting from a configuration obtained in the course of these previous numerical simulations and carefully putting up the two parallel hard walls, the systems of hard helical particles with $r=0.2 D$ and $p=4 D$ too were relatively promptly seen to develop a twist of their local nematic director, that is, they were transforming to state points within a $\mathrm{N}_{c}^{*}$ phase, as the relevant functions $g_{2}^{\widehat{\mathbf{u}}}(x)$ finally indicate (Fig. 7). Both the sign and the order of magnitude of the corresponding $\mathcal{P}$ are found in agreement with theoretical calculations, though reaching a reasonable stationary value for the still negative $q$ required lengthier runs than in the case of systems of hard helical particles with $r=0.2 D$ and $p=9.92 D$. With respect to the latter hard helical particle systems (Fig. 5), the functions $g_{1, \Delta}^{\widehat{\mathrm{w}}, \widehat{\mathrm{u}}}\left(r_{/}\right)$nonetheless reveal an incipient screw-like ordering (Fig. 7) suggesting that it could further develop at larger values of $\mathrm{P} D^{3} / k_{B} \mathrm{~T}$. This is already the case at $\mathrm{P} D^{3} / k_{B} \mathrm{~T}=0.7$ and 0.8 where the functions $g_{1, \Delta}^{\widehat{\mathbf{w}}, \widehat{\mathbf{u}}}\left(r_{/ /}\right)$show an undamped cosinusoidal form [Figs. 8(a) and 8(c)], in accord with previous numerical simulations employing 3D-PBC that assigned these two state points to a $\mathrm{N}_{s}^{*}$ phase. Rather than starting from a screw-like nematic configuration previously obtained at the same value of dimensionless pressure, these numerical simulations were deliberately started from the same nematic configuration from which the present run at $\mathrm{P} D^{3} / k_{B} \mathrm{~T}=0.6$ (Fig. 7) did start. If the behaviour of $g_{1, \Delta}^{\widehat{\mathbf{w}}, \widehat{\mathbf{u}}}\left(r_{/ /}\right)$thus proves that screw-like ordering has spontaneously developed even once the system had been confined between two parallel hard walls, the corresponding $\theta$ profiles [Figs. 8(b) and 8(d)] are not flat. Rather, at both these values of dimensionless pressure, the system appears as having ended up blocked into configurations characterised by two, overall flat, extremal regions with a noticeable screw-like ordering, separated and tied by an intermediate region of variable thickness in which $\theta(x)$ accordingly drops and the degree of screw-like ordering reduces [Figs. 8(b), 8(d), and 8(e)]. One natural interpretation of these results is that each extremal region corresponds to a screw-like nematic film wetting the respective hard wall. The further growing of these two films into one single domain could be impeded for two alternative reasons. Either the intermediate region corresponds to an inbulk thermodynamically stable $\mathrm{N}_{c}^{*}$ phase and the two, overall flat, extremal regions are nothing more than screw-like nematic films wetting the respective hard wall or the $\mathrm{N}_{s}^{*}$ phase is the in-bulk thermodynamically stable phase and the two extremal region misalignment takes very long to heal. To help resolve this doubt, we resorted to the MD method to exploit its capability of naturally dealing with the system constituent particle collective motion. Starting from a cholesteric configuration previously obtained at $\mathrm{P} D^{3} / k_{B} \mathrm{~T}=0.6$, a lengthy MD-NPT calculation was carried out on a system of soft-repulsive helical particles confined between two parallel soft-repulsive walls at
$\mathrm{P} D^{3} / k_{B} \mathrm{~T}=0.8$. The system loses its initial cholesteric character after $\sim 2 \times 10^{6}$ time steps and, by forming also a central region with an overall flat $\theta(x)$ profile, apparently progresses towards a single-domain untwisted screw-like nematic configuration; after $320 \times 10^{6}$ time steps, this situation is so nearly reached that one may confidently state that a neat $\mathrm{N}_{s}^{*}$ phase is indeed forming starting from a cholesteric configuration (Fig. 9). The latter untwisting process was eventually observed also at $\mathrm{P} D^{3} / k_{B} \mathrm{~T}=0.9$ in a system of hard helical particles confined between two parallel hard walls. Starting from a cholesteric configuration taken from the early stage of the run at $\mathrm{P} D^{3} / k_{B} \mathrm{~T}=0.7$ and then letting the system evolve [Figs. 10(a) and 10(b)], a complete spontaneous untwisting of the local nematic director was finally detected [Fig. 10(c)]. This final phase is a screw-like (weakly) smectic A as determined by calculating the smectic order parameter ${ }^{39} \tau=0.25$, suitable pair correlation functions, as well as confirmed by direct visualisation [Figs. 10(d) and 10(e)]. The same results
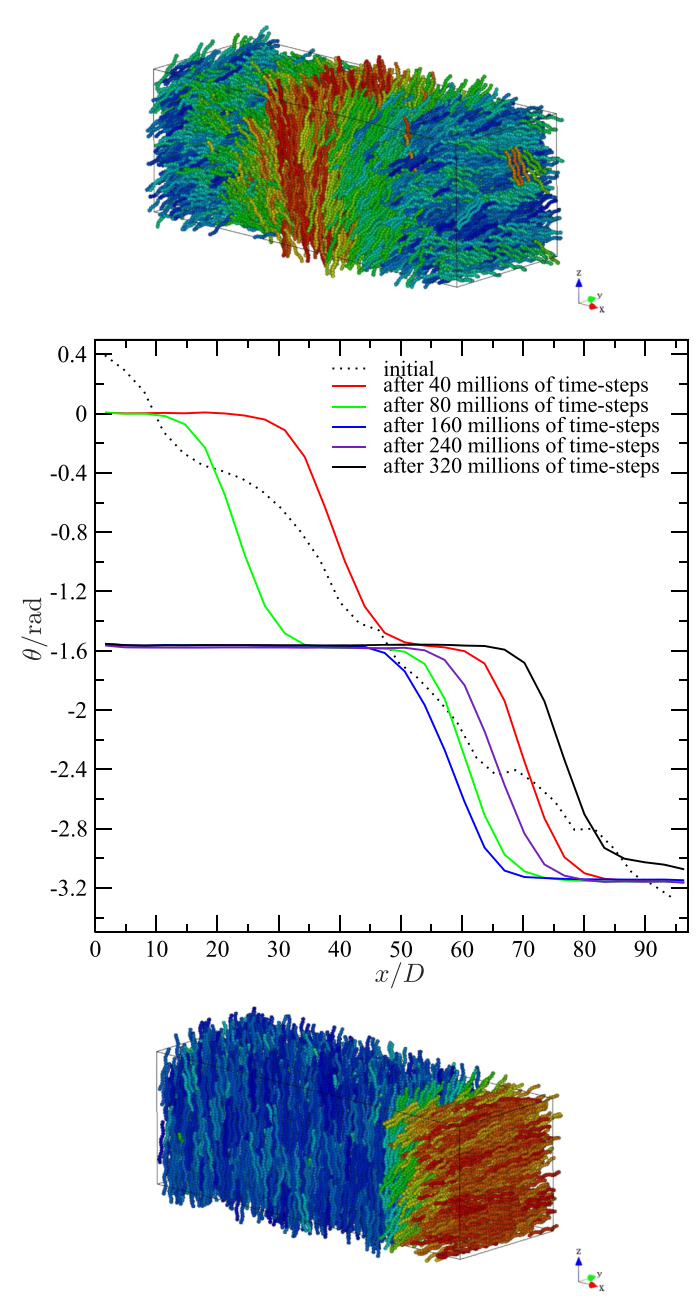

FIG. 9. Evolution of the local nematic director $\theta$ as a function of $x$ at several moments of the MD-NPT calculation carried out on a system of soft-repulsive helical particles with $r=0.2 D$ and $p=4 D$; apart from the initial, each profile is an average over $10 \times 10^{6}$ of time steps the final instant contributing to this average being that one indicated in the label. Images ${ }^{37}$ are shown of the initial (top) and final (bottom) configuration. The soft-repulsive helical particles are coloured according to the angle that their $\widehat{\mathbf{u}}$ axes forms with the eigenvector corresponding to the largest eigenvalue of the global nematic order matrix. ${ }^{35}$ 

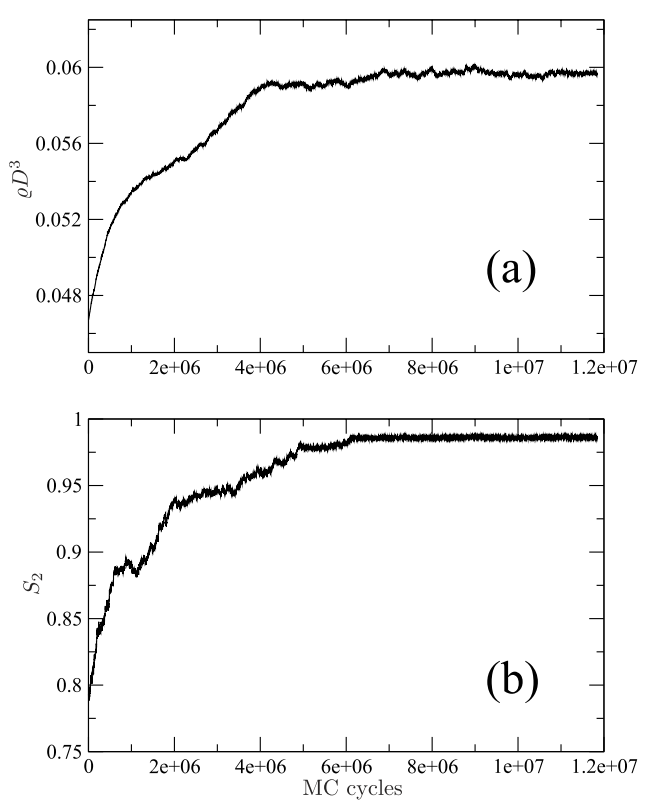
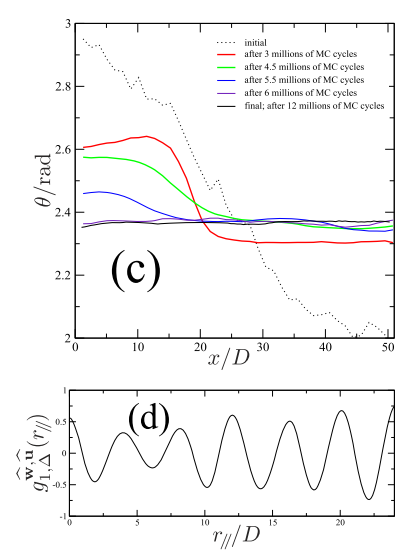

(e)

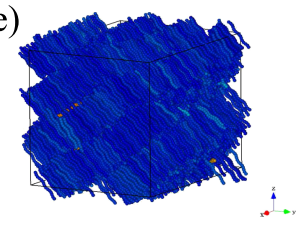

FIG. 10. Evolution of dimensionless number density (a) and nematic order parameter (b) as a function of MC cycles in a system of hard helical particles with $r=0.2 D$ and $p=4 D$ starting from a cholesteric configuration at $\mathrm{P} D^{3} / k_{B} \mathrm{~T}=0.9$; (c) local nematic director angle $\theta$ as a function of $x$ at several "instants" of MC "time" during the same calculation of (a) and (b); (d) pair correlation function $g_{1, \Delta}^{\widehat{\mathbf{w}}, \widehat{\mathbf{u}}}(r / /) ;(\mathrm{e})$ image $^{37}$ of the system of hard helical particles with $r=0.2 D$ and $p=4 D$ in the screw-like smectic A phase as seen from the $y z$ plane normal; observe the layered structure in which these particles self-assemble and glimpse a very few of them lying in the inter-layer regions transverse to the layer normal. The hard helical particles are coloured according to the angle that their $\widehat{\mathbf{u}}$ axes forms with the eigenvector corresponding to the largest eigenvalue of the global nematic order matrix. ${ }^{35}$ were obtained starting from a moderately dense orthorhombic lattice in which the hard helical particles were perfectly aligned along the $z$ axis; the only differences being that
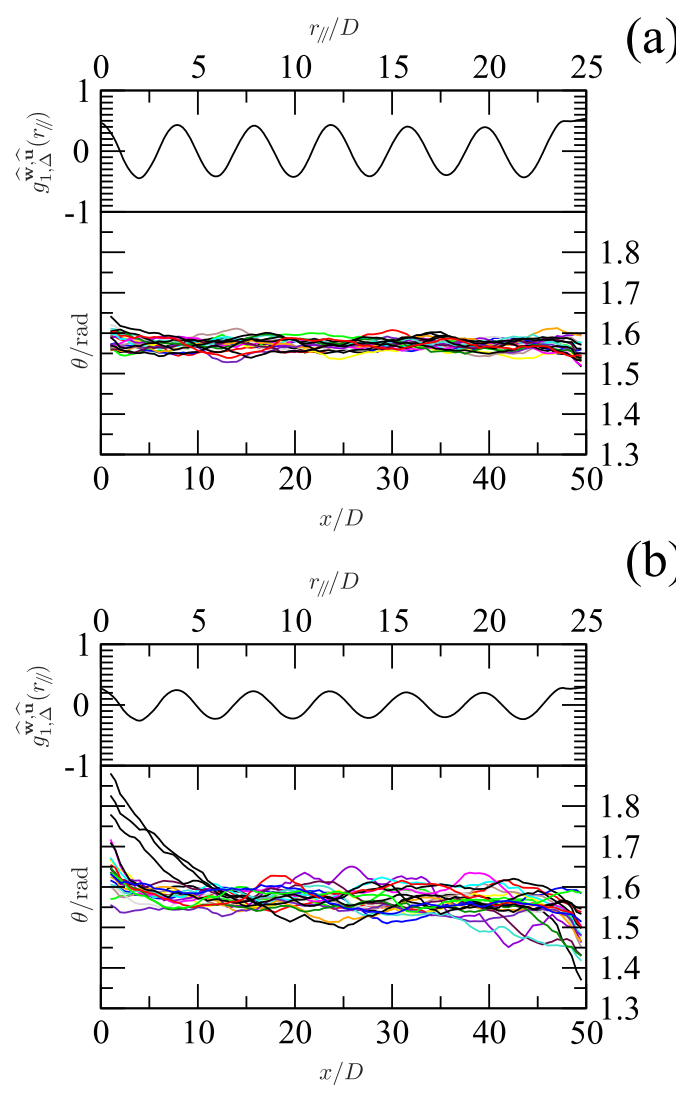

FIG. 11. Pair correlation functions $g_{1, \Delta}^{\widehat{\mathbf{w}}, \widehat{\mathbf{u}}}\left(r_{/ /}\right)$(top panel) and the angle $\theta$ that the local nematic director forms with the $y$ axis as a function of $x$ (bottom panel) for a system of hard helical particles with $r=0.2 D$ and $p=4 D$ as obtained in $\mathrm{MC}$ numerical simulations starting from a configuration obtained at $\mathrm{P} D^{3} / k_{B} \mathrm{~T}=0.9$, in its turn, obtained at the end of a run started from a moderately dense orthorhombic lattice configuration inserted between two parallel hard walls at $\mathrm{P} D^{3} / k_{B} \mathrm{~T}=0.8$ (a) and 0.7 (b). The various coloured thin full curves shown in the bottom panel of (a) and (b) are each an average over 1000, one-thousand-MC-cycle separated, configurations. reaching the equilibrium state was significantly faster and the degree of smectic ordering was larger $(\tau=0.67)$. These findings are consistent with those previously obtained at the same dimensionless pressure by employing 3D-PBC. ${ }^{6,7}$ Starting from a screw-like smectic A configuration obtained in this latter run, additional MC-NPT calculations were carried out at $\mathrm{P} D^{3} / k_{B} \mathrm{~T}=0.8,0.7$, and 0.6 . The flatness of the $\theta$ profile, the waviness of the function $g_{1, \Delta}^{\widehat{\mathbf{w}}, \widehat{\mathbf{u}}}\left(r_{/ /}\right)$, and the small value of $\tau$ $=0.09$ are all indicative of a single-domain $\mathrm{N}_{s}^{*}$ phase at $\mathrm{PD}^{3} / k_{B} \mathrm{~T}=0.8$ [Fig. 11(a)]. The same considerations would also hold at $\mathrm{PD}^{3} / k_{B} \mathrm{~T}=0.7$ were it not for those fringes now visible at either hard walls; a symptom that the $\mathrm{N}_{c}^{*}$ phase is close to forming [Fig. 11(b)]. In fact, it does at $\mathrm{PD}^{3} / k_{B} \mathrm{~T}=0.6$, consistent with the results also obtained by starting from a nematic configuration inserted between the two parallel hard walls (Fig. 7).

\section{CONCLUSIONS}

The aim of the present study was to address two important points originated from our past work on hard helical particle system. First, the observability of the cholesteric phase; (elongated) hard helical particles are expected to form a cholesteric phase, but its pitch can be orders of magnitude larger than usual computational box dimensions and thus a proper cholesteric twist can remain unobservable using three-dimensional periodic boundary conditions. Second, the stability of the screwlike nematic phase, a novel chiral nematic phase special to helical particles that we observed in past work, against a proper cholesteric phase.

By using isobaric-isothermal Monte Carlo and molecular dynamics methods, we have studied systems of helical particles confined between two parallel repulsive walls so as to give a proper cholesteric phase the possibility to form. Our findings confirm the existence of the two chiral nematic phases and are tersely summarised as

(I) for weakly curly helical particles $(r=0.2 D$ and $p=9.92 D)$, we observe a cholesteric phase. 
(II) In the opposite limit of relatively curly helical particles ( $r=0.4 D$ and $p=4 D$ ), we find a screw-like nematic phase and no evidence of the cholesteric phase.

(III) In the intermediate case $(r=0.2 D$ and $p=4 D)$, both the cholesteric and the screw-like nematic phases are present at, respectively, lower and higher densities.

In principle, both cholesteric and screw-like nematic phases can exist for a system of helical particles, but the particle morphology controls the relative stability of these phases so that, for a given system, either may be absent. For this reason, phase diagrams and trends obtained without taking into account both cholesteric and screw-like nematic phases should be taken with caution.

The features of the cholesteric phase, when present, are in general agreement with current Onsager-like theory results. For $r=0.2 D$, with both $p=4 D$ and $p=9.92 D$, a lefthanded cholesteric phase is observed, as predicted by Straley ${ }^{1}$ for right-handed helical particles with geometric parameters similar to those under study. Besides handedness, the Onsagerlike theory calculations ${ }^{5,7,11-13}$ provide reasonable estimates of the magnitude of the cholesteric pitch, of the order of 100-200D, which slightly decreases on going from $D=9.92$ to $D=4$. The decrease of the pitch with increasing density found for the case $r=0.2 D$ and $p=9.92 D$ is also in agreement with the theoretical calculations for $r=0.2 D$ and $p=8 D$ in Ref. 7. Indeed, a decrease of the cholesteric pitch, i.e., an increase of phase chirality, with increasing density is generally predicted for hard helical with $p / D$ values larger than a few units. In Refs. 12 and 13, the opposite trend, i.e., an increase of the cholesteric pitch with increasing density, is reported for both $r=0.4 D$ and $p=4 D$ and $r=0.2 D$ and $p=4 D$. However, such theoretical predictions refer to density ranges where the cholesteric phase does not seem to exist. Indeed, our numerical simulations do not show any cholesteric phase for the former helical particles, whereas for the latter, the cholesteric range is so restricted that it is admittedly hard to identify a trend for the pitch as a function of density. In both these cases, we have found the screw-like nematic phase instead. ${ }^{4,6,7}$

The theoretical calculations for the cholesteric phase presented thus far ${ }^{5,7,11-13}$ cannot account for screw-like nematic ordering. There is a need for a more general approach, as outlined in Ref. 40, to describe both chiral nematic phases.

Our present evidence suggests that the two chiral nematic phases are mutually exclusive. However, we cannot fully exclude that cholesteric and truly long-ranged screw-like orderings might find a way to indeed coexist (e.g., via the analog of the twist-grain-boundary smectic phase ${ }^{41}$ ).

Note added in proof: Following the acceptance of our manuscript and its ensuing posting on the arXiv, Randall Kamien (University of Pennsylvania) kindly pointed out to us that his theory published in J. Phys. II France, ${ }^{42}$ originally dealing with the effects of a competition between cholesteric and hexatic bond-orientational orderings in a nematic phase, may be more generally re-interpreted as relevant to a nematic phase where the cholesteric ordering competes with any ordering that, like screw-like ordering, perpendicularly twists round a fixed nematic director; in this light, our results appear to support this theory and we are grateful to him for this comment.

\section{ACKNOWLEDGMENTS}

G.C. thanks the Government of Spain for the award of a Ramonv́ y Cajal research fellowship and acknowledges its financial support under Grant Nos. FIS2013-47350-C5-1-R and MDM-2014-0377. This work was also supported by Italy MIUR via No. PRIN-2010-2011 programme under Grant No. 2010LKE4CC. A.F. thanks C. Greco for the setup of the molecular dynamics numerical simulations.

${ }^{1}$ J. P. Straley, Phys. Rev. A 14, 1835 (1976).

${ }^{2}$ P. G. de Gennes and J. Prost, The Physics of Liquid Crystals (Clarendon Press, Oxford, 1993).

${ }^{3}$ E. Frezza, A. Ferrarini, H. B. Kolli, A. Giacometti, and G. Cinacchi, J. Chem. Phys. 138, 164906 (2013).

${ }^{4}$ H. B. Kolli, E. Frezza, G. Cinacchi, A. Ferrarini, A. Giacometti, T. S. Hudson, J. Chem. Phys. 140, 081101 (2014).

${ }^{5}$ E. Frezza, A. Ferrarini, H. B. Kolli, A. Giacometti, and G. Cinacchi, Phys. Chem. Chem. Phys. 16, 16225 (2014).

${ }^{6}$ H. B. Kolli, E. Frezza, G. Cinacchi, A. Ferrarini, A. Giacometti, T. S. Hudson, C. De Michele, and F. Sciortino, Soft Matter 10, 8171 (2014).

${ }^{7}$ H. B. Kolli, G. Cinacchi, A. Ferrarini, and A. Giacometti, Faraday Discuss. 186, 171 (2016).

${ }^{8}$ E. Barry, Z. Hensel, Z. Dogic, M. Shribak, and R. Oldenbourg, Phys. Rev. Lett. 96, 018305 (2006).

${ }^{9}$ F. Manna, V. Lorman, R. Podgornik, and B. Zeks, Phys. Rev. E 75, 030901(R) (2007).

${ }^{10}$ On this point, cf. the numerical simulation study by R. Memmer, J. Chem. Phys. 114, 8210 (2001).

${ }^{11}$ S. Belli, S. Dussi, M. Dijkstra, and R. van Roij, Phys. Rev. E 90, 020503(R) (2014).

${ }^{12}$ S. Dussi, S. Belli, R. van Roij, and M. Dijkstra, J. Chem. Phys. 142, 074905 (2015).

${ }^{13}$ M. M. C. Tortora and J. P. K. Doye, J. Chem. Phys. 146, 184504 (2017).

${ }^{14}$ The common tenet that the thermodynamic stability of a $\mathrm{N}_{c}^{*}$ phase is very close to that of a $\mathrm{N}$ phase providing $\mathcal{P}$ is sufficiently large ${ }^{2}$ and motivated the usage of an Onsager-like (density functional) theory, ${ }^{15}, 16$ within the implementation originally developed by Straley, ${ }^{1}$ to explore the characteristics of the $\mathrm{N}_{c}^{*}$ phase, in particular, its pitch handedness and value in systems of hard helical particles. ${ }^{5,7}$ These results, then reproduced also by others either using a different model ${ }^{17}$ or the same model and a different implementation of the same theory, ${ }^{11-13}$ were useful in that they confirmed that phenomena such as cholesteric handedness inversion, often explained by invoking a competition between different energetic and entropic terms that would promote opposite phase chirality, may arise also in pure systems of suitable hard chiral particles; in such cases, they are solely entropy driven. One may nonetheless note that cholesteric pitch handedness inversion, while possibly "dramatic" if viewed from the pitch side, is a more ordinary phenomenon if viewed from the corresponding wavevector side; it merely means that the wavevector versus density function has a zero at a certain density value and behaves linearly in its neighbourhood.

${ }^{15}$ L. Onsager, Ann. N. Y. Acad. Sci. 51, 627 (1949).

${ }^{16}$ J. D. Parsons, Phys. Rev. A 19, 1225 (1979); S. D. Lee, J. Chem. Phys. 87, 4972 (1987); 89, 7036 (1988).

${ }^{17}$ H. H. Wensink, Europhys. Lett. 107, 36001 (2014).

${ }^{18}$ On this point, cf. G. Kumaraswamy et al., Faraday Discuss. 186, 371 (2016).

${ }^{19}$ D. Frenkel, Eur. Phys. J. Plus 128, 10 (2013).

${ }^{20}$ M. Melle, M. Theile, C. K. Hall, and M. Schoen, Int. J. Mol. Sci. 14, 17584 (2013).

${ }^{21}$ S. Dussi and M. Dijkstra, Nat. Commun. 7, 11175 (2016).

${ }^{22}$ Š. Růžička and H. H. Wensink, Soft Matter 12, 5205 (2016).

${ }^{23}$ The results presented in this work were preliminarily shown as part of an oral communication entitled "Self-assembly of hard helical particles: cholesteric and screw-like, nematic and smectic, phases" given at the XII International Conference on Materials Chemistry, 20-23 July 2015, York, England. 
${ }^{24}$ G. Cinacchi, A. M. Pintus, and A. Tani, J. Chem. Phys. 145, 134903 (2016).

25 J. D. Weeks, D. Chandler, and H. C. Andersen, J. Chem. Phys. 54, 5237 (1971).

${ }^{26}$ W. M. Saslow, M. Gabay, and W. M. Zhang, Phys. Rev. Lett. 68, 3627 (1992).

${ }^{27}$ The method of self-determined spiralling boundary conditions has been applied in the numerical simulation of on-lattice cholesteric models: R. Memmer and F. Janssen, J. Chem. Soc., Faraday Trans. 94, 267 (1998); Liq. Cryst. 24, 805 (1998); Z. Naturforsch., A 54, 747 (1999); Mol. Phys. 101, 1829 (2003). One has to note that (b) and (d) already showed that a temperature-induced cholesteric pitch handedness inversion occurs in pure systems of spins interacting via a simple pair potential energy function that, apart from a non-chiral part, is composed of a single chiral part, without the need to invoke different energetic contributions each one promoting opposite phase chirality: i.e., that the cholesteric pitch handedness depends not only on the interparticle potential energy of interaction but also on the thermodynamic state of the system.

${ }^{28}$ N. Metropolis, A. W. Rosenbluth, M. N. Rosenbluth, A. H. Teller, and E. Teller, J. Chem. Phys. 21, 1087 (1953).

${ }^{29}$ B. J. Alder and T. E. Wainwright, J. Chem. Phys. 27, 1208 (1957); A. Rahman, Phys. Rev. 136, A405 (1964).

${ }^{30}$ W. W. Wood, J. Chem. Phys. 48, 415 (1968); 52, 729 (1970).

${ }^{31}$ S. Nosé, J. Chem. Phys. 81, 511 (1984); W. G. Hoover, Phys. Rev. A 31, 1695 (1985).
${ }^{32}$ M. P. Allen and D. J. Tildesley, Computer Simulation of Liquids (Clarendon Press, Oxford, 1987); D. Frenkel and B. Smit, Understanding Molecular Simulation (Academic Press, San Diego, 2002); W. Knauth, Statistical Mechanics: Algorithms and Computations (Oxford University Press, Oxford, 2006).

${ }^{33}$ S. Plimpton, J. Comput. Phys. 117, 1 (1995); http://lammps.sandia.gov.

${ }^{34}$ H. Kamberaj, R. J. Low, and M. P. Neal, J. Chem. Phys. 122, 224114 (2005).

${ }^{35}$ J. Vieilliard-Baron, Mol. Phys. 28, 809 (1974).

${ }^{36}$ H. H. Wensink and L. Morales-Anda, J. Chem. Phys. 143, 144907 (2015).

${ }^{37}$ A. T. Gabriel, T. Meyer, and G. Germano, J. Chem. Theory Comput. 4, 468 (2008); http://qmga.sourceforge.net.

${ }^{38}$ On this point, cf. also the results obtained for systems of hard twisted triangular prisms. ${ }^{21}$

${ }^{39}$ See for example P. J. Wojtowicz, in Introduction to Liquid Crystals, edited by E. B. Priestley, P. J. Wojtowicz, and P. Sheng (Plenum Press, New York, 1975), Chap. 7.

${ }^{40}$ G. Cinacchi, A. Ferrarini, E. Frezza, A. Giacometti, and H. B. Kolli, in Self-Assembling Systems: Theory and Simulation, edited by L. T. Yan (Wiley, Chichester, 2017).

${ }^{41}$ P. G. de Gennes, Solid State Commun. 10, 753 (1972); S. R. Renn and T. C. Lubensky, Phys. Rev. A 38, 2132 (1988); J. W. Goodby, M. A. Waugh, S. M. Stein, E. Chin, R. Pindak, and J. S. Patel, Nature 337, 449 (1989).

${ }^{42}$ R. Kamien, J. Phys. II France 6, 461 (1996). 